\title{
Development of hypothalamic obesity in the male golden hamster (Mesocricetus auratus) as a function of food preference
}

\author{
H. E. MARKS* and C. R. MILLER†,†† \\ University of Georgia, Athens, Ga. 30601
}

When fed a preferred diet, male hamsters with lesions of the ventromedial hypothalamus gained reliably more weight than control animals. There were no reliable differences in weight gain between lesioned and control groups when the diet was nonpreferred. In addition, lesioned hamsters differed from control animals in nest-building behavior and open-field activity, independent of weight differences. The groups could not be differentiated on the basis of reactivity to capture and handling. It was suggested that hypothalamic obesity in the male golden hamster is due, at least in part, to dietary preferences. Other behavioral changes resulting from lesions of the ventromedial hypothalamus may only be peripherally related to food-motivated behaviors and may be a function of the species-typical behaviors of the particular species studied.

Previous attempts to demonstrate obesity following lesions of the ventromedial hypothalamus (VMH) in the golden hamster have generally been unsuccessful. Matalka (1967) and Seago (unpublished study) found no evidence of increased weight gain in male hamsters as a result of $\mathrm{VMH}$ lesions. Cox (unpublished study) reported obesity in some animals, but a large proportion of hamsters died.

Animals with VMH lesions exhibit altered taste preferences (Corbit \& Stellar, 1964; Graff \& Stellar, 1962; Marks \& Brown, 1971). Matalka (1967) and Seago limited the diet of their hamsters to standard lab chow. Although Cox supplemented the hamster's diet with sunflower seeds, the supplement was insufficient to represent a significant portion of the diet. The lack of success in producing hypothalamic obesity may be attributable to the lab chow diet, since lab chow is a nonpreferred food for hamsters.

The present study attempted to produce hypothalamic obesity in hamsters by providing them with constant access to sunflower seeds in addition to lab chow. In addition to measures of weight gain, a number of other behavioral measures were made in order to evaluate the similarity of the effects of VMH lesions in hamsters and rats.

*The first author was supported by NIMH Postdoctoral Fellowship MH-48317 during this study.

+Now at the Department of Psychology Georgia College at Milledgeville, Milledgeville, Ga. 31061 .

$\dagger+\mathrm{T}$ he authors would like to express their sincere appreciation to Dr. B. N. Bunnell for his interest and numerous helpful suggestions throughout this study. The authors would also like to thank Dr. J. D Seago for his assistance in obtaining the coordinates for surgery.

\section{METHODS}

Twenty-eight adult male hamsters (Mesocricetus auratus), ranging in weight from 123 to $171 \mathrm{~g}$, were used. The animals were housed individually with ad lib access to food and water. Animals were provided with strips of paper toweling for nesting materials.

Lesions were made by passing a direct current of $1.0 \mathrm{~mA}$ through the uninsulated tip of the electrode for 15 $(N=6)$ or $20 \sec (N=8)$. An operated control group was subjected to the same surgical procedures, but no lesions were made $(\mathrm{N}=6)$. The stereotaxic coordinates were computed from instrument zero using a leveled skull: $\mathrm{AP}=+3.8 \mathrm{~mm}$, $\mathrm{L}=0.6 \mathrm{~mm}$, and $\mathrm{DV}=0.7 \mathrm{~mm}$ above the base of the skull. An additional eight hamsters served as an unoperated control group.

Half of each lesion and each control group $(\mathrm{N}=14)$ received sunflower seeds in addition to Purina Lab Chow throughout the experiment. The other half of the animals $(\mathrm{N}=14)$ received only lab chow for the first 5 weeks postoperatively, after which sunflower seeds were added to their diet for the remainder of the study.

The animals were weighed every 3 days for 15 days and then weekly until the end of the study. Food intake measures could not be made since the hamsters receiving sunflower seeds hid some in their nests and threw some out of the cage onto the floor.

Measures of resistance to capture and reactivity to handling were made prior to weighing the hamsters (Table 1). The quality of nest construction was rated before the animals were handled. The rating scale for nest construction was the same as that used by Matalka (1967; Table 2).

Open-field activity was measured 1, 5 , and 9 weeks postoperatively. The apparatus was an open field, 36 in. square, enclosed by 18-in.-high walls. The walls and floor of the apparatus were painted white. Black lines divided the floor into 169 -in. squares. Each hamster was started from the same one of the four center squares and observed for $3 \mathrm{~min}$ per trial. Two measures were taken: latency to leave the start square and number of squares entered during the trial. A square was counted as entered if the hamster placed at least two feet into the square. Each hamster received one trial per day for 3 days during each of the three testing periods.

At the conclusion of the study, the hamsters were sacrificed with an overdose of sodium pentobarbital and profused with $0.09 \%$ saline. The brains were fixed in formalin and embedded in celloidon. Thirty-micron sections were taken through the area of the lesion and stained with thionin for histological verification.

$$
\text { RESULTS }
$$

Two lesioned hamsters died following surgery. Eight of the remaining 12 animals became obese and were found to have bilateral lesions destroying most if not all of the ventromedial nucleus of the hy pothalamus (VMN) and immediately surrounding tissue. The histology of the lesioned nonobese hamsters indicated that two had lesions destroying small portions of the VMN but extending into the lateral hypothalamic area; one received only a unilateral lesion; and the last animal had bilateral damage to the posterior portion of the VMN, but the

Table 1

Rating Scale for Resistance to Capture and Reactivity to Handling

1 Hamster does not resist capture and remains quiet while held.

2 Hamster makes minimal resistance to capture and shows small amount of movement while being held.

3 Hamster resists capture by moving away and moves vigorously while being held.

4 Hamster resists capture by running away and struggles very vigorously while being held.

5 Hamster resists capture by attacking the gloved hand and struggles vigorously while being held.

Table 2

Rating Scale for the Quality of Nest Construction

0 Paper scattered over cage floor.

1 Paper accumulated toward rear of cage.

2 Pallet in rear of cage but considerable paper still scattered.

3 Pallet, but ragged about the edges.

4 Pallet, edges neat, no paper scattered.

5 Pallet, sides against rear and side wall(s).

6 Nest with sides all around.

7 Nest with sides and top. 


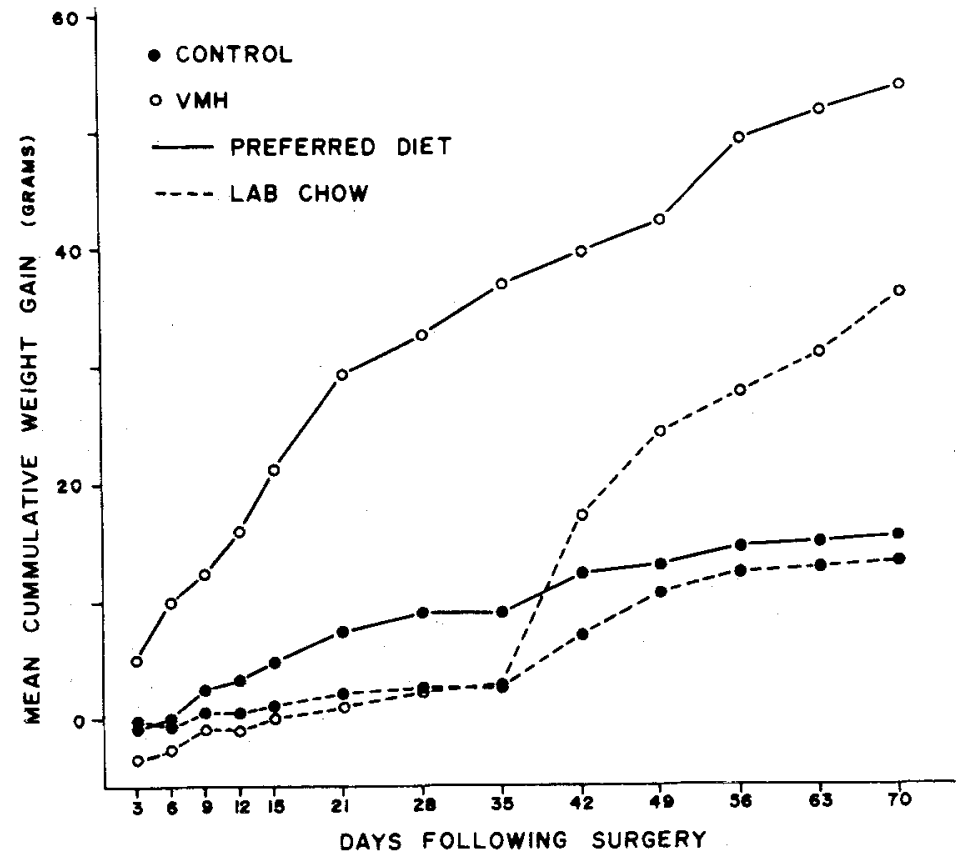

Fig. 1. Mean increase in weight during the 70 days following surgery for all groups.

lesion extended well into the posterior hypothalamic area. These nonobese lesioned animals were omitted from the statistical analyses.

Lesions of $1.0 \mathrm{~mA}$ for $20 \mathrm{sec}$ produced slightly larger lesions than did $1.0 \mathrm{~mA}$ for $15 \mathrm{sec}$. The former group did not gain reliably more weight than the latter and no differences in behavior could be attributed to the differences in current duration. No statistical distinctions were made between the groups.

Hamsters given sunflower seeds gained more weight than those restricted to lab chow $(p<.05)$. Lesioned hamsters fed sunflower seeds outgained control groups fed the same diet $(p<.01)$. No reliable differences in weight were found between lesioned and control groups during the 5 weeks they were maintained solely on lab chow (Fig. 1). When these groups were given access to sunflower seeds, all animals increased their rate of weight gain $(p<.05)$, but the lesioned group gained more than the control groups $(\mathrm{p}<.05)$

Lesioned hamsters apparently restricted their diet almost exclusively to sunflower seeds, while control animals ate lab chow as well as seeds. Since accurate food intake measures could not be made, the results are based on the number of times animals of either group required lab chow during the postoperative period. No lesioned hamster required additional lab chow during the time the animals had access to both lab chow and sunflower seeds. Each control animal that weight, 200+-g hamsters are not uncommon.

At no time during the study could any of the groups be differentiated on the basis of resistance to capture or reactivity to handling. The only hamster which gave any indication of viciousness was a lesioned hamster that died 4 days later ( 7 days postoperatively).

Regardless of the diet received, lesioned hamsters made reliably less adequate nests than did the control hamsters $(p<.05)$, although nest quality did improve over time for the lesioned animals $(p<.05)$. Lesioned hamsters fed only lab chow appeared to build somewhat poorer nests than those fed sunflower seeds, but these differences did not reach statistical significance and disappeared completely after the lab chow group was given access to sunflower seeds (Fig. 2).

Lesioned hamsters did not differ reliably from control hamsters in the number of squares entered over all trials, but were reliably slower to leave the start square $(p<.05)$. There were no reliable differences among the groups as a function of the diet they received (Fig. 3 ). The lesioned hamsters had a smaller reduction in the number of squares entered over the three testing periods than did the control animals $(p<.05)$, and, while the control groups increased their latency to leave the start square, the lesioned group showed a decrease in latency over weeks $(p<.05)$.

\section{DISCUSSION}

Obesity can be produced in 


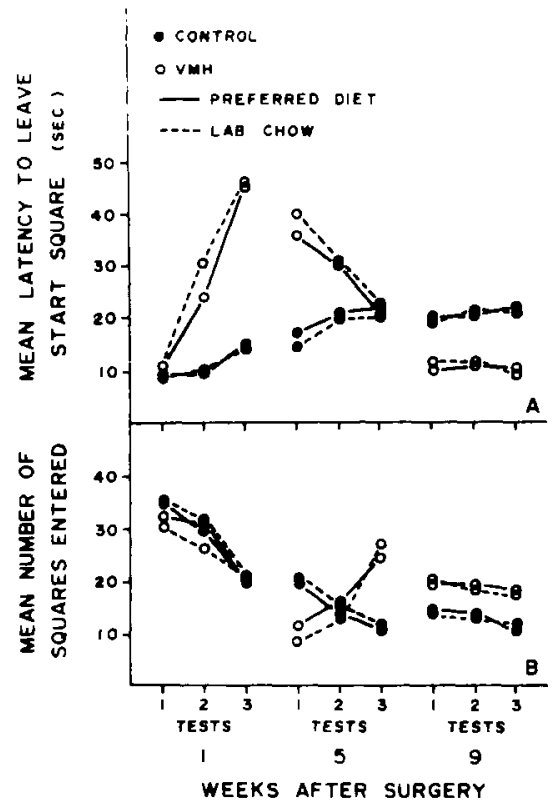

Fig. 3. Open-field activity. (A) Latency to leave the start square. (B) Number of squares entered during the $3-\mathrm{min}$ trial.

hamsters with VMH lesions, but the effects of the lesion appear to be dependent upon the hamsters' preference for the particular food(s) provided. Lesioned hamsters demonstrate the same type of increased weight gain observed in other species when they are allowed access to a preferred food. With a less-preferred diet, no reliable weight gain was seen.

The viciousness associated with VMH lesions in the rat (Marks \& Brown, 1971; Stevenson, 1969) was not observed in the VMH hamster. This lack of lesion-induced viciousness may be a function of the species-typical modes of response of the hamster, since a lack of viciousness has also been reported in the septal lesioned hamster (Sodetz \& Bunnell, 1967; Sodetz et al, 1967), while septal rats almost invariably display some degree of "septal rage" (Kondo \& Lorens, 1971; Turner, 1970). This lack of viciousness following VMH lesioning has also been observed in the gerbil (Brown \& Marks, unpublished data).
The fact that the VMH hamster demonstrates nest construction deficits regardless of its weight level (present study) and normal weight hamsters have food hoarding deficits (Matalka, 1967) suggests that the $\mathrm{VMH}$, at least in the hamster, may be involved with functions which are only peripherally related to food-motivated behaviors. The range of these other functions and the extent to which they are characteristic of the species used is, at present, unknown.

The open-field data suggests a number of possible interpretations. Lesioned hamsters may initially be more reactive to the stimuli associated with the open field and, therefore, decrease their latency to leave the start square more slowly than control hamsters. This explanation would not account for the increase in latency from Trial 1 to Trial 3 during the first week's tests. A conclusion of lesion-induced hyperactivity would also be premature since the greater overall activity of the lesioned hamsters would be explained equally well by a failure to habituate to the open field. Activity measures for VMH-lesioned hamsters need to be replicated with other measuring devices so that a broader picture of activity changes can be examined and hamster activity can be directly compared to the activity data derived from VMH-lesioned rats.

With one exception, weight increases appear to be a consistent result of VMH damage over a broad spectrum of the phylogenetic scale, from man (Reeves \& Plum, 1969) to birds (Kuenzel \& Helms, 1970). The only exception to this conclusion is a study using guinea pigs (Joseph \& Knigge, 1968). They were not able to demonstrate increased weight gain following VMH lesions in either male or female guinea pigs. Their guinea pigs were fed a commercial lab chow which may have been nutritionally adequate but less preferred than fresh green feeds and hay, their natural diet (Ibsen, 1950; Woodnott, 1969). If the same logic applied to hamsters and rats can be applied to guinea pigs, the negative results of the guinea pig study may be a function of a nonpreferred diet rather than a lack of effectiveness of the VMH lesions.

While weight increases are a consistent correlate of VMH lesions, there are a number of other effects (hoarding, activity, nest building, viciousness) which may depend upon the particular species-typical behavior patterns of the animal studied. Research with VMH-lesion effects have concentrated the bulk of its effort on the areas of food intake and food-motivated behavior in the rat so that evidence for species-typical behavioral changes as a function of VMH lesions is limited.

CORBIT, J. DEFERENCS Palatability, food intake, and obesity in normal and hyperphagic rats. Journal of Comparative Physiology, 1964, 58, 63-67.

GRAFF, H., \& STELLAR, E. Hyperphagia obesity, and finickiness. Journal of Comparative \& Physiological Psychology, $1962,55,418-424$

IBSEN, H. L. The guinea pig. In E. J. Farris (Ed.), The care and breeding of laboratory animals. New York: Wiley, 1950.

JOSEPH, S. A., \& KNIGGE, K. M. Effects of VMH lesions in adult and newborn guinea pigs. Neuroendocrinology, 1968 , 3, 309-331.

KUENZEL, W. J., \& HELMS, C. W. H y perphagia, polydipsia, and other effects of hypothalamic lesions in the white - thraoted sparrow. Zontrichia Albicollis. Cond or, 1970, 72, 66-75.

KONDO, C. Y., \& LORENS, S. A. Sex differences in the effects of septal lesions. Physiology \& Behavior, 1971, 6, 481-485.

MARKS, H. E., \& BROWN, G. E. The effects of VMH lesions in Charles River rats. Psychonomic Science, 1971, 23. 117-119.

MATALKA, E. S. The hoarding behavior and food intake of the hamster following hypothalamic and limbic forebrain lesions. Unpublished doctoral dissertation, University of Florida, 1967.

REEVES, A. G., \& PLUM, F. Hyperphagia, rage, and dementia accompanying a ventromedial hypothalamic neoplasm. Archives of Neurology, 1969, 20, 616-624.

SODETZ, F. J., \& BUNNELL, B. N. Septal ablation and the social behavior of the golden hamster. Paper read at Midwestern Psy chological Association, 1967.

SODETZ, F. J., MATALKA, E. S., \& BUNNELL, B. N. Septal ablation and affective behavior in the golden hamster. Psychonomic Science, 1967, 7, 189-190.

STEVENSON, J. A. F. Mechanisms in the control of food and water intake. Annals of the New York Academy of Sciences, $1969,157,1069-1083$.

TURNER, B. H. Neural structures involved in the rage syndrome of the rat. Journal of Comparative \& Physiological Psychology, 1970, 7, 103-113.

WOODNOTT, D. P. Nutrition. In $D$. J. Short and D. P. Woodnott (Eds.), The I.A.T manual of laboratory animal practice and techniques. Springfield, Ill: Charles C Thomas, 1969. 